\title{
Ecuador, los inmigrantes y la propaganda fascista $(1922-1938)^{*}$
}

\author{
por
}

\section{Chiara Pagnotta ${ }^{1}$}

Taller de Estudios e Investigaciones Andino-Amazónicos, Universidad de Barcelona

En este texto se analiza el tema de la propaganda fascista en Ecuador, dirigida por un lado a la opinión pública y al gobierno ecuatoriano y por otro al grupo italiano que vivía en Ecuador en el periodo que va, desde la toma del poder en Italia por parte de Mussolini (1922), hasta la víspera de la segunda guerra mundial (1938). El objetivo de este artículo es analizar cuestiones relativas a la inmigración proveniente de un país totalitario, tratando de abordar dos nudos: en primer lugar, el de la relación entre el fascismo y la sociedad ecuatoriana, y en segundo lugar el de los mecanismos de consenso que se impulsan a través de la propaganda, su actividad y sus logros o fracasos entre los inmigrantes italianos, y entre la élite y la sociedad ecuatoriana de la época. El estudio se apoyará en la documentación proveniente, en su mayoría, del Archivio Storico Diplomatico de Roma y del Archivo Histórico Alfredo Pareja Diezcanseco del Ministerio de Relaciones Exteriores de Quito.

PALABRAS ClaVE: Ecuador; Italia; fascismo; propaganda; inmigrantes; sociedad ecuatoriana.

Cómo citar este artículo / Citation: Pagnotta, Chiara, "Ecuador, los inmigrantes y la propaganda fascista (1922-1938)", Revista de Indias, LXXX/279 (Madrid, 2020): 481-508. https://doi.org/10.3989/revindias.2020.014.

\section{INTRODUCCIÓN}

Reporta el historiador Angelo Trento que, en ocasión de la exposición por los diez años de la nombrada «revolución fascista», hubo un pabellón dedi-

* Este trabajo se enmarca en el proyecto de investigación I+D+i HAR2015-64891-P (MINECO/FEDER, UE) que se ha desarrollado en el seno del TEIAA (2014SGR532), grupo de investigación consolidado por el Comissionat per a Universitats i Recerca del DIUE de la Generalitat de Catalunya.

1 chiara.pagnotta@ub.edu, ORCID iD: https://orcid.org/0000-0002-9810-3109 
cado a los «italianos en el exterior» cuyo lema era una frase pronunciada por Mussolini: «dovunque è un italiano, là è il tricolore, là é la patria, là è la difesa del Governo» (en cualquier lugar en el que haya un italiano, ahí está la bandera, ahí está la patria, ahí está la defensa del Gobierno)².

Las políticas migratorias del fascismo se diferenciaron radicalmente de las anteriores, liberales, hacia finales de los años veinte, cuando la emigración hacia el exterior pasó a representar para el régimen una dispersión de las fuerzas humanas que Italia necesitaba para transformarse en potencia mundial. Cabe subrayar que los esfuerzos fascistas por transformar a los inmigrantes en instrumentos útiles al régimen iban de la mano con el objetivo de limitar la emigración, pues ambas tendencias estaban dirigidas a recuperar las fuerzas italianas para las batallas que Italia estaba combatiendo ${ }^{3}$.

Destacamos que, según las prácticas y el lenguaje del régimen fascista, no existían los inmigrantes italianos, existían más bien italianos en el exterior que hacían grande la madre patria ${ }^{4}$. Ellos eran, por ende, el útil instrumento geopolítico del colonialismo italiano en los territorios en los que vivían y habían esparcido la supuesta grandeza italiana fuera de Italia ${ }^{5}$.

Precisamente el mantenimiento y fortalecimiento del ligamen entre inmigrantes y madre patria fue uno de los rasgos que caracterizaron el periodo fascista. El historiador Luconi resalta además, para el caso estadunidense, de qué manera, en su política de relaciones exteriores, Mussolini actuó también gracias a una Diplomazia parallela, que incluía la utilización de los inmigrantes italianos como grupo de presión, para influir en la orientación de los gobiernos extranjeros en aquellos países en los que residían ${ }^{6}$. Además, las tentativas de esta diplomacia estaban dirigidas a promover en el exterior una imagen positiva de Italia de la época, presentándola como una potencia innovadora. El fin era ostentar el régimen de Mussolini como elemento de progreso en relación con la larga decadencia de los gobiernos liberales anteriores.

Cabe subrayar que Ecuador nunca ha sido un destino privilegiado para la emigración italiana. En todo caso, la importancia del grupo italiano destaca cuando, en 1899, se lleva a cabo un «Censo piloto», limitado a la ciudad de Guayaquil, y este certifica que, frente a 51.115 ecuatorianos, había 9369 extranjeros: 5368 peruanos, 1318 colombianos, 642 chinos, 640 italianos y 296

2 Trento, 2005: 3. Las traducciones del italiano al español son de la autora.

3 Bertonha, 2001a: 43. Pretelli, 2004: 50.

4 Ostuni, 2001: 317-319.

5 Sobre la teoría de un imperialismo pacífico, véase entre otros Bertonha, 2001b. Trento, 2005. Luconi y Tintori, 2004. Franzina y Sanfilippo, 2003.

6 Luconi, 2000. 
españoles, entre otros ${ }^{7}$. Luego, en el primer Censo nacional de 1950, consta que en Ecuador residen 23.489 extranjeros. Los primeros tres países transoceánicos de procedencia son Alemania (933 inmigrantes), Italia (884 inmigrantes) y España (616 inmigrantes $)^{8}$.

Por parte de Ecuador, vemos cómo las políticas orientadas a incrementar la inmigración extranjera y europea se implementan a partir de 1895, bajo los primeros gobiernos liberales. La inmigración europea, según las ideas de las élites de poder, debía estar compuesta predominantemente por trabajadores agrícolas que pudieran enseñar a los locales los métodos de la agricultura basada en la pequeña propiedad (de tipo familiar, en colonias agrícolas), para incorporar al país al mercado mundial en calidad de exportador ${ }^{9}$.

Conforme a lo afirmado por Ayala Mora, bajo el régimen liberal ecuatoriano se pusieron las bases para el control del estado por los grupos burgueses, y la vinculación con el sistema internacional ${ }^{10}$. La transformación de Ecuador en país exportador supuso un crecimiento de las inversiones de capitales por parte principalmente de los países extranjeros, y entre ellos principalmente de Gran Bretaña, seguida por Alemania, Francia e Italia. Además, era notorio que, en Ecuador, entre finales del siglo XIX y las primeras décadas del XX, los inmigrantes alemanes e italianos controlaban la distribución de gran parte de las importaciones que llegaban a Guayaquil del exterior, teniendo así en las manos la economía del principal puerto de Ecuador. Varios de entre esos exitosos comerciantes extranjeros lograron rápidamente ser parte de la élite de la sociedad ecuatoriana ${ }^{11}$.

Sabemos que, tras la primera guerra mundial, a finales de los años veinte, aconteció una depresión económica. Concretamente esto significó la caída de los precios del cacao y de la entera economía ecuatoriana que dependía de las exportaciones. La crisis económica vino seguida de la del sistema político que aquella provocó, y que desembocó en el golpe de Estado protagonizado por los llamados «militares jóvenes», en julio de 1925, los cuales apartaron del poder al presidente Córdova. Luego sucedió la crisis económica global de 1929, todo lo cual ayudó al primer triunfo electoral de Velasco Ibarra $(1933)^{12}$.

\footnotetext{
7 «Censo 1899», en Roca, 1900: anexo sin número de página.

8 Ministerio de Economía - Dirección General de Estadística y Censos, 1950: Tabla ${ }^{\circ} 35$ a.

9 Pagnotta, 2016: 77-80. La pepita de cacao era en aquel entonces el principal producto de exportación.

10 Ayala Mora, 2008: 96.

11 Crawford, 1980: 108-109.

12 Para un trabajo de síntesis acerca de la historia ecuatoriana del periodo, véase, entre otros, Ayala Mora, 1983; Quintero y Silva, 2001, tomo I.
} 
A nivel geopolítico, el Ecuador post-independiente estaba buscando nuevas y diferentes relaciones internacionales respecto a España. Desde un punto de vista económico hay que subrayar que durante la primera guerra mundial el país andino había re-orientado gran parte de sus exportaciones de cacao, de Gran Bretaña y Alemania, hacia Estados Unidos ${ }^{13}$. A finales de 1915, los Estados Unidos cubrían el $45 \%$ de todo el comercio de importación de Ecuador, mientras en 1911 eran el $27 \%{ }^{14}$.

Durante la primera guerra mundial, Ecuador y otros países del área declararon primero su neutralidad en el conflicto, y luego rompieron las relaciones con Alemania y se acercaron a Estados Unidos. Exactamente al final de la guerra, conforme a lo pasado en toda Sudamérica, se fue imponiendo la hegemonía económico-comercial de Estados Unidos a costa de los otros países occidentales, y particularmente de Gran Bretaña.

Desde el punto de vista historiográfico, las relaciones entre inmigrantes, nacionalismos y patria de origen están hoy en día alcanzando una importancia hasta ahora desconocida dentro de los estudios sobre migraciones. Precisamente las conexiones entre inmigración, nacionalismo y construcción del Estado Nación en América Latina son un novedoso campo de estudio hoy en día todavía poco indagado ${ }^{15}$. De otro lado, en el campo de investigación sobre el fascismo, la propaganda y la proyección internacional ${ }^{16}$, juegan un papel particular los trabajos de Federico Finchelstein, quien, a partir de sus investigaciones sobre el contexto argentino, llega a estudiar el fascismo como ideología política trasnacional y global, poniendo énfasis en los intercambios entre las sociedades involucradas ${ }^{17}$.

La presencia de los grupos extranjeros en Ecuador es un tema que hasta ahora ha sido poco estudiado por la historiografía ecuatorianista. Una de las razones es, por un lado, que los inmigrantes provenientes de países no limítrofes han sido siempre numéricamente poco relevantes, a pesar de la importancia que tuvieron los italianos, los asiáticos, los alemanes y los españoles, entre otros, para la historia de la sociedad ecuatoriana. Por otro lado, es cierto que durante mucho tiempo la sociedad ecuatoriana se imaginó como una sociedad prevalentemente blanco-mestiza, de origen europeo, cuya alte-

13 Quintero y Silva, 2001, tomo I: 277.

14 Crawford, 1980: 159-160.

15 Se distinguen las contribuciones recientes de Foote y Goebel, 2014. Lesser, 2013. FitzGerald y Cook-Martín, 2014. Yankelevích, 2015.

${ }^{16} \mathrm{La}$ producción académica en este ámbito es muy amplia. Entre otros, es importante destacar el trabajo fundamental de Emilio Gentile, 1995.

17 Véase en particular Finchelstein, 2010. 
ridad era representada no por los inmigrantes europeos, sino más bien por los grupos indígenas. Solo a partir de los años noventa, también a consecuencia de los grandes levantamientos indígenas, el país tuvo que confrontarse y asumir sus varias raíces étnicas. En este sentido, los estudios históricos y antropológicos que siguieron han contribuido a implementar el conocimiento sobre los grupos que han poblado el país y su historia migratoria ${ }^{18}$.

Situándose en la línea de estudios sobre migraciones trasnacionales y construcción de las identidades nacionales en América Latina, este artículo propone estudiar la actuación del grupo (o de los grupos) italiano(s) en Ecuador como parte de la historia del país andino entre guerras, tratando de desvelar la influencia y el papel que el fascismo y los fascistas tuvieron en la sociedad de acogida. Además, interesa estudiar el ejemplo de una diáspora en América Latina y su relación con el país de origen y con el de llegada. Precisamente, se pretende trabajar acerca de la inmigración proveniente de un país totalitario, para ver los mecanismos de consenso que se impulsan a través de la propaganda, su actividad y sus logros o fracasos entre los inmigrantes italianos, y entre la élite y, más en general, la sociedad ecuatoriana de la época.

En términos metodológicos, se ha intentado sostener una perspectiva dirigida por un lado a las relaciones internacionales y geopolíticas - en las que los inmigrantes son entendidos como los elementos humanos de esa relación entre estados-, y por otro a enfocar cómo este proceso histórico era vivido por los actores sociales del periodo.

Es oportuno destacar que el tema ha sido poco trabajado hasta ahora, a causa de la dificultad de acceder a la documentación de la época. Por parte italiana, es sabido que la gran dificultad en la reconstrucción de la acción exterior del fascismo se debe a que buena parte del material producido por la dirección central de los fasci en el exterior, y por las diferentes secciones en los varios países, está desaparecido o perdido ${ }^{19}$. De otro lado, por parte ecuatoriana, varios historiadores y archiveros han señalado la incuria y la escasez del patrimonio documental del país, en particular por cuanto concierne a los fondos del siglo XIX y la primera parte del siglo XX. El mal estado de conservación hace que, además, parte de la documentación del periodo sea a menudo fragmentaria ${ }^{20}$.

18 Sin pretensión de ser exhaustiva: Almeida, 1997. Bejarano, 1997. Crawford, 1996. Di Capua, 1998. Embajada de Italia (Quito), 2008. Estrada, 1992; 1993. Grubel Rosenthal, 2010. Guarnieri Calò Carducci, 2001. Kreuter, 1997. Pagnotta, 2016. Ramírez, 2012.

19 Gentile, 1995: 898.

20 Entre otros, se señalan Bustos, 2011. Saint Geours, 1994: 185. 
En la primera parte de este texto se aborda el tema de la propaganda fascista dirigida, prevalentemente, a la opinión pública y al gobierno ecuatoriano, y sus efectos en el país andino. Aquí el texto se centrará en tres momentos clave para entender cómo esta propaganda se declina en influencia: se mostrarán los gérmenes del imperialismo pacífico en la Italia liberal, la llegada a Ecuador del buque Italia, en 1924, y la actuación del país durante la invasión italiana de Etiopia (1935-1936). Luego, el texto se centrará en mostrar la obra de propaganda dirigida más específicamente a la sociedad civil ecuatoriana y a los italianos en Ecuador. También, en ese caso, algunos elementos serán claves para entender los logros (o no) de la propaganda: la publicación de la revista La idea latina, las relaciones en el seno de la sociedad 'Garibaldi' y, finalmente, el proyecto de entrega de un monumento a Ecuador como homenaje a la amistad entre los dos países. En la conclusión, se ofrecerán algunas observaciones de carácter general a partir del estudio del caso aquí dibujado.

\section{DOVUNQUE ל̀ UN ITALIANO...}

Durante los primeros años en el poder, las políticas fascistas en torno a la inmigración no se alejaron mucho de las del estado liberal italiano ${ }^{21}$; ambas trataron de utilizar a los inmigrantes como instrumento geopolítico. Ya desde finales del siglo XIX, en el seno de la Società Geografica Italiana, el debate entre americanistas y africanistas representó una contraposición entre quienes privilegiaban el ámbito mediterráneo para la expansión militar italiana, y quienes preferían una penetración económica y comercial al otro lado del Atlántico. Para los últimos, se hacían cada vez más evidentes las relaciones entre colonialismo, expansionismo y emigración, hechos que dejaban entrever las posibilidades de una colonización pacífica, que podía llevarse a cabo gracias a la presencia de los emigrantes en los territorios americanos ${ }^{22}$. Parece evidente que, en cuanto a postura ideológica, el fascismo, en su fase inicial, recuperó - entre otras - estas ideas de colonización pacífica ${ }^{23}$ de los territorios latinoamericanos, y que el proyecto era llevarla a cabo mediante el comercio, la cultura y la emigración.

Fue después de 1926 cuando, tras la aprobación de las leggi fascistissime y la transformación en estado totalitario, se abolió el Commissariato

\footnotetext{
21 Bianchi, 1994.

22 Para una síntesis sobre este argumento, véase Bertonha, 2001a. Furfaro, 1982.

23 Se imaginaba pacífica debido a la falta de medios económicos y militares que padecía Italia de la época.
} 
Generale dell'immigrazione (1927) y quedó limitada la libertad de emigrar. Esto rindió manifiesto que, para el régimen, la emigración hacia el exterior representara ahora una inútil dispersión de fuerzas, que tenían que ser dirigidas hacia la colonización interna de la península italiana y hacia los territorios del naciente imperio ${ }^{24}$. En lugar del Commissariato se creó una $\mathrm{Di}$ rezione Generale degli Italiani all'Estero, dependiente del Ministerio de Relaciones Exteriores.

Esto, sin embargo, no significó una disminución del interés del gobierno por los italianos en el exterior. Afirma el historiador Emilio Gentile, que entre los objetivos del fascismo estuvo el de fascistizzare a los emigrantes y transformarlos en instrumentos de su ideología 25 . El canal principal fueron los Fasci italiani en el exterior, nacidos como organización del Partido fascista, y transferidos a las dependencias del Ministerio de Relaciones Exteriores en 192726 .

El papel que jugaron las organizaciones de los fasci en el exterior se fue definiendo poco a poco. Por un lado, el mismo Mussolini, en el discurso de clausura del primer congreso de la organización en 1925, clamó por la disciplina y por la obediencia tanto a las leyes de los países de llegada (para evitar alteraciones y tensiones en las relaciones diplomáticas con otros países) como a las representaciones diplomáticas del país de origen. Luego, según el nuevo estatuto escrito en 1929 por el mismo duce, la organización de los fasci estuvo privada de toda libertad política y de acción, y estuvo englobada en una estructura centralizada, a cuya cabeza estaban las representaciones consulares ${ }^{27}$. A partir de este momento fue clarísimo que el papel de los fas$c i$ era el de hacer propaganda y difusión de los ideales fascistas en las sociedades huéspedes, y, entre ellas, la ecuatoriana. Es evidente que el interés de Mussolini estaba dirigido a actuar sobre el extranjero, con el fin de ampliar la simpatía por parte de los otros gobiernos hacia las políticas fascistas ${ }^{28}$.

Por otra parte, se destaca cómo las relaciones económico-comerciales privilegiadas entre Ecuador e Italia habían empezado antes, en la década de los años veinte, y terminaron en vísperas de la segunda guerra mundial. Conforme a lo afirmado por Soave, para Ecuador, la atracción (económica, comercial,

24 Mas en general, sobre la política del fascismo en relación a la emigración véase también: Cannistraro y Rosoli, 1979.

25 Gentile, 1995: 897.

26 Véase Franzina y Sanfilippo, 2003.

27 De Caprariis, 2003: 13-18.

28 Entre otros, véase Bertonha, 1999; 2001a; 2001b para Brasil; Ciccarelli, 1988 para Perú. 
cultural y militar) que, antes de la primera guerra mundial, ejercía Alemania, había disminuido a causa de su derrota en dicha guerra, y había sido remplazada por las tentativas de influencia que procuraban ejercer otros países occidentales (Estados Unidos, Gran Bretaña, España, Francia e Italia) ${ }^{29}$. Realmente, para evitar una dependencia excesiva respecto a los Estados Unidos, el país andino trató de establecer colaboraciones y acuerdos comerciales con otros países, incluyendo a Italia ${ }^{30}$.

Cabe subrayar que, por parte italiana, en la búsqueda de estas relaciones (económicas) privilegiadas entre la Italia fascista y Ecuador, jugó un papel importante la idea de una común latinidad, el panlatinismo, que se contraponía tanto al panamericanismo de matriz estadunidense, como a la idea de la hispanidad que proponía la antigua madre patria España. La idea de latinidad proponía la comunión de la gran familia latina, basada en afinidades históricas, culturales y religiosas (es decir, espirituales, según la propaganda de la época). A la cabeza de la latinidad se encontraría Italia, heredera de la antigua Roma e, implícitamente, en un rol subalterno, todos los otros países «latinos» ${ }^{31}$.

Ya en 1919, la Italia liberal había encargado al coronel Benedetto Accorsi una gira para tantear a las diferentes repúblicas americanas acerca de las posibilidades de desarrollar allá planes económico-comerciales. Por lo que concierne al caso ecuatoriano, en 1921 los resultados de aquel viaje se concretaron en la creación de la Compagnia Italiana Dell'Ecuador (CIDE) - financiada con capitales privados (por parte, entre otros, del mayor banco italiano de la época, la Banca Commerciale Italiana) - y con el patrocinio público $^{32}$. La CIDE estaba encargada de promover actividades financieras, industriales, agrícolas y militares.

Otro resultado del viaje de Accorsi fue la llegada de la Misión Militar italiana, con la encomienda de la reorganización de las fuerzas armadas del país andino ${ }^{33}$. Eso se debe a que Ecuador, entonces, en relación con otros países latinoamericanos, estaba intentando modernizar su aparato bélico, para

29 Soave, 2008: 57.

30 Ibidem: 24 et seg.

31 Scarzanella y Trento, 2004: 225-226. Los debates sobre la idea de la latinidad y el papel de los grupos de inmigrantes italianos en el extranjero son numerosos. Entre otros, véase Poupalt, 2009. Pretelli, 2008.

32 Más en detalle en Guarnieri Calò Carducci, 2001: 49 et seg. Pagnotta, 2016, 113-119. Soave, 2008: 51-90.

33 En aquel entonces, la Misión estaba compuesta por 18 oficiales, algunos suboficiales (todos ellos excombatientes en la primera guerra mundial) y algunos civiles con funciones técnicas. 
alcanzar los niveles que los países europeos habían logrado en la primera guerra mundial.

La tentativa italiana parecía ser la de posicionarse en primer lugar, respecto a otras naciones europeas - en particular Alemania, ahora dañada por la derrota militar en la primera guerra mundial—, como socia económica de Ecuador.

Algunos de los logros más evidentes de la Misión militar italiana fueron el desarrollo del sistema de aviación local, y la compraventa de armas italianas para abastecer al ejército del país andino. Además, se estipularon toda una serie de acuerdos con la finalidad de permitir a los oficiales ecuatorianos - de diferentes cuerpos de la armada- completar sus entrenamientos en escuelas italianas ${ }^{34}$.

Se hace constar que, bajo la presidencia de Tamayo (1920-1924), las actividades italianas en Ecuador tuvieron un notable impulso porque, según la diplomacia del país europeo, el presidente buscaba alejarse de la influencia política chilena y frenar el influjo estadounidense ${ }^{35}$.

La llegada al poder de Mussolini no cambió las relaciones ni los acuerdos entre los dos países, aunque, ya en 1923, el jefe del gobierno italiano clamó por un redimensionamiento del contingente militar presente en el país, que no estaba, en su opinión, desarrollando ninguna función útil. La situación fue peor para la CIDE, que siguió en sus labores, a pesar de haber perdido cada apoyo político. Esas dinámicas marcaron el paso de un interés económico-colonial, típico de la época liberal, a uno político-propagandístico, más en sintonía con los intereses fascistas ${ }^{36}$.

\section{La Real Nave Italia en Ecuador}

Junto con la misión militar, otro legado de la época liberal que los fascistas hicieron suyo, concretaron y volcaron como instrumento de propaganda del régimen, fue la gira de la Real Nave Italia en América Latina, que, finalmente, tuvo lugar en 1924.

34 Véanse varios documentos en el Archivo Histórico del Ministerio de Relaciones Exteriores de la República de Ecuador (AHMRE), N.37.33, Comunicaciones dirigidas Misiones Diplomáticas del Extranjero (1925).

35 Riassunto delle situazione politica della Repubblica dell'Ecuatore (1924-1929), Archivio Storico Diplomatico del Ministero degli Affari Esteri (ASDMAE), Serie Affari Politici 1919-1930, pacco 1014 Equatore (1927-1930).

36 Soave, 2008: 95. 
Los fascistas dieron al viaje un significado político, tratando de transformarlo en una obra de propaganda en favor propio, y de reforzamiento de los ligámenes entre italianos en el exterior y la madre patria fascista. El irredentista dannunziano, nacionalista y luego fascista, y finalmente ministro, Giovanni Giurati, fue nombrado embajador extraordinario del Rey de Italia para la gira ${ }^{37}$.

El viaje del buque, que atracó en los principales puertos del continente, tenía como objetivos el de promover las relaciones comerciales, el de buscar nuevos socios económicos en América Latina, y el de fortalecer los vínculos con los grupos italianos en el continente. La idea del gobierno italiano era que el buque exhibiera las mejores producciones de Italia de la época e inspirara en los inmigrantes «el legítimo orgullo de sentirse hijos de la gran madre de la Latinidad» ${ }^{38}$. Conforme a lo afirmado por Moure Cecchini, el crucero se propuso coadyuvar en la realización de un imperialismo cultural antes que militar, basado en las afinidades culturales e históricas entre la madre patria de la latinidad y los países americanos ${ }^{39}$. La idea de la cercanía cultural política y económica, bajo el común denominador de la idea de latinidad, entre Italia e América Latina, aparecía claramente como la motivación del crucero, tal como afirma el representante ecuatoriano en su informe anual:

El objeto de la travesía de la Real Nave 'Italia' es el de hacer conocer a las Repúblicas de la América Latina, habitadas por pueblos muy semejantes al nuestro y donde viven y prosperan millones de italianos, el alto grado de desarrollo y perfeccionamiento alcanzado por las actividades industriales y comerciales italianas posteriores a la Guerra, y de sentar así sólidas bases para el anhelado incremento de las relaciones económicas, culturales y políticas entre Italia y sus hermanas latinas $^{40}$.

La nave tenía un espacio para exposiciones de 3000 metros cuadrados, dos aéreos, una estación de telégrafo, una exposición de artistas italianos de la época (con pinturas, estatuas y otras obras artísticas) y la dotación estaba

37 Giovanni Giurati combatió en la primera guerra mundial, a causa de una herida tuvo que abandonar la línea del frente. Fue exponente del grupo de los nacionalistas al interior del fascismo. Participó de la empresa de Fiume (1919) junto con Gabriele D'annunzio. Después de la marcia su Roma (1922) entró al Gobierno Mussolini, en calidad de ministro delle terre liberate y presidente del Consiglio Superiore dell'Emigrazione.

38 La Real Legación de Italia. "El Viaje del buque Italia”, AHMRE, B.25.2, Legación de Italia en el Ecuador (1921-1925).

39 Moure Cecchini, 2016.

40 Ponce, 1924: 206. 
compuesta por 400 hombres entre emprendedores, intelectuales, periodistas y artistas, a cuya cabeza estaba Giovanni Giurati ${ }^{41}$. La idea era exponer las producciones nacionales italianas y la cultura de vanguardia de la península, haciendo hincapié en el potencial técnico, comercial, agrícola, industrial y artístico del país. Destacan las palabras que podemos leer en el periódico de orientación liberal, El Telégrafo de Guayaquil sobre el buque: «Es una magnífica muestra del valer de esta nación admirable, que se alza pujante, después de la horrorosa guerra que asaltó a Europa» ${ }^{42}$.

El 10 de junio, mientras el buque estaba por entrar al océano Pacifico, fue secuestrado el diputado socialista Giacomo Matteotti, quien había denunciado en el parlamento italiano los fraudes y las violencias ocurridas en las elecciones del 6 de abril, que habían dado lugar al triunfo del Partido Nacional Fascista (PNF) sobre los otros partidos. También por esa razón, cuando el buque atracó en Chile, hubo disturbios, y protestas en Valparaíso y Santiago, y la prensa de Antofagasta publicó unos artículos de crítica a la gira ${ }^{43}$. La nave, en su expedición, atracó en Guayaquil el 3 de agosto, etapa que se desarrolló pacíficamente.

De la minuciosa descripción que hacen los periódicos ecuatorianos, en particular El Telégrafo de Guayaquil, se puede entender que la etapa ecuatoriana fue un éxito a nivel publicitario. Además, dicho periódico publicó un dosier dedicado a la Italia contemporánea, del que se deduce una simpatía hacia el fascismo, en cuanto régimen que había dado orden al caos y devuelto a Italia a su antiguo esplendor [en referencia a la antigua Roma] ${ }^{44}$.

El 3 de agosto, el buque entró triunfalmente en el puerto de Guayaquil, donde muchos ecuatorianos habían ido a esperar su llegada. Acompañaron la entrada al puerto el embajador italiano y el general Pirzio Biroli, en aquel entonces jefe de la misión militar, recién subidos a bordo ${ }^{45}$.

Para la llegada al puerto había sido creado un comité organizador de bienvenida. Formaban parte de él varios exponentes de la élite ecuatoriana ${ }^{46}$; y

41 Algunos pasajeros escribieron libros y memorias del evento. Giovanni Giurati escribió una relación del viaje; una síntesis de la tapa ecuatoriana basada en dicha fuente se puede leer en Guarnieri Calò Carducci, 2001: 42-45.

42 "El próximo arribo del buque «Italia»", El Telégrafo, 8 de julio de 1924.

43 Moure Cecchini, 2016: 470.

44 En particular véase: "La real nave Italia en el Ecuador", El Telégrafo, 3 de agosto de 1924 y más en general todo el dosier publicado como anexo el mismo día.

45 "El imponente arribo de la regia nave exposición Italia al puerto de Guayaquil", El Telégrafo, 4 de agosto de 1924.

46 Incluyendo Carlos Arroyo del Rio. Fuente: "El próximo arribo de la real nave «Italia»", El Telégrafo, 17 de julio de 1924. 
otro que había surgido, más específicamente italiano, el Comité Patriótico Italiano, del que formaban parte los más destacados del grupo ${ }^{47}$.

Los navegantes fueron agasajados con varios eventos oficiales, y se organizaron diversos momentos de encuentro y celebración: bailes en su honor, banquetes, proyecciones cinematográficas del ejército y de la marina italianas, conferencias y exposiciones sobre el oriente ecuatoriano y sobre los salesianos, entretenimiento dramático en el colegio salesiano Cristóbal Colon, conferencias y congreso de importadores y exportadores promovido por la Cámara de Comercio de Guayaquil, entre otros, además de las varias visitas de diferentes grupos a bordo del buque. Destaca cómo los sindicados universitarios y obreros habían procedido a elegir a sus representantes para las visitas al buque. Entre otros productos exhibidos a bordo, las ametralladoras producidas por la fábrica FIAT fueron puestas a prueba en un «ejercicio» ${ }^{48}$, evidenciando las intenciones de la gira, en realidad no muy escondidas, de encontrar mercados para las producciones italianas, incluyendo las bélicas. Un momento particularmente solemne de la estadía en Guayaquil fue la entrega de una urna que contenía la tierra «sagrada» del Carso (territorio del frente italiano en la primera guerra mundial) a los ex combatientes italianos que habían luego emigrado a Ecuador ${ }^{49}$. Nos consta, además, que, precisamente en ocasión del viaje del buque, en el que se pudo aprovechar la presencia del ministro Giurati, se procedió a la inauguración de la sección ${ }^{50}$ local del Fascio que había sido fundada el mes de junio del mismo año.

Otro comité se formó para que parte de los tripulantes viajaran a Quito para la bienvenida y para visitar la ciudad, los monumentos y las obras coloniales ${ }^{51}$. En ocasión de esa visita, se publicó una guía turístico-cultural de la 1924.

47 "La nave Italia fondeo en Puna ayer a las 2 y 20 p.m.", El Telégrafo, 3 de agosto de

48 "La estadía de los marinos italianos en el puerto. Las pruebas de las ametralladoras Fiat", El Universo, 9 de agosto de 1924.

49 Varios artículos de $E l$ Telégrafo entre 2 de agosto y el 8 de agosto de 1924.

${ }^{50}$ La sección fue fundada por iniciativa de Luigi Fossati, que era en aquel entonces administrador encargado del Fascio para Ecuador. En el momento de su fundación, la sede de Guayaquil contaba con 40 inscritos. En la ciudad de Manta existía otra sección del Fascio. Desconocemos otras informaciones al respecto. Aliprandi y Martini, 1930a: [sin número de páginas]. En 1933 los inscritos al fascio de Guayaquil eran 82 en total. Aliprandi y Martini, 1930b: [sin número de páginas]. En todo caso, los fasci en Ecuador no parecen destacar por su importancia y su actuación. Según lo afirmado por el cónsul italiano en 1930: «Es continuado en este trimestre [de 1930] el completo estancamiento». Al Ministero degli Affari Esteri, Roma, Il Regio Console Gino Busi, Maggio 28 1930, ASDMAE, Serie Affari Politici 1919-1930 pacco 1014 Equatore (1927-1930), prot. n 1394 A1, A. VIII.

51 "La visita del buque Italia", El Universo, 21 de junio de 1924. 
ciudad de Quito ${ }^{52}$. Además, Giovanni Giurati fue recibido en Quito por el presidente Luis Tamayo ${ }^{53}$.

Finalmente, el cadáver del diputado Matteotti fue encontrado en un bosque, en las afueras de Roma, el 16 de agosto, cuando el buque Italia ya había dejado Ecuador y estaba por entrar en el Caribe.

\section{ECUADOR, ITALIA Y LAS SANCIONES}

La agresión italiana a Etiopia fue otro elemento que desveló la compleja trama de las relaciones internacionales entre el régimen fascista y los países americanos, y el esfuerzo de propaganda del fascismo a las elites locales. Cabe destacar que finalmente Ecuador entró a formar parte de la Sociedad de las Naciones el 27 de septiembre de $1934{ }^{54}$. A partir de septiembre de 1935 fue elegido Miembro no permanente del Consejo de la Sociedad ${ }^{55}$, y con este rol tuvo que enfrentar la guerra ítalo-etiópica. El papel que jugó el país andino no deja de dar luz a las ambigüedades con las que se enfrentó a este conflicto, en el que Italia, un país «amigo», y socio económico importante en aquella época, figuraba como agresor.

Para contrastar precisamente esta acusación de agresión, Italia concentró todos sus esfuerzos propagandísticos en presentarse como víctima de las brutalidades etíopes, y a la vez como salvadora de las poblaciones subyugadas por los mismos etíopes. Incluso antes de la invasión, en el mes de agosto de 1935, en el memorándum que presenta el ministro de la Legación de Italia al ministro de Relaciones Exteriores de Ecuador, Italia se identificaba y presentaba frente al ministro ecuatoriano como la primera nación en llevar la «civilización» a las tierras etíopes, y destaca las históricas tentativas de agresión a las colonias italianas en el área, por parte de los etíopes, mientras Italia estaba ocupada en otros asuntos (como la ocupación de Libia entre 1911 y 1912, la primera guerra mundial, o las más recientes operaciones militares de «pacificación» en Somalia). El memorando italiano matiza además cómo Etiopía estaba desarrollando un programa de equipamiento bélico. Italia clamaba que era necesario tomar medidas para garantizar la seguridad de sus colonias, además de presentar al país africano como el que

52 La nave 'Italia' en el Ecuador. Recuerdo de la visita de la Embajada italiana y su séquito. La muy noble y muy leal ciudad de San Francisco de Quito - Agosto 1924, 1924.

53 Ponce, 1924: 206.

54 Ponce Borja, 1935: 198 et seg.

55 Chiriboga, 1936: 61. 
había violado los acuerdos pacíficos previos. La tentativa evidente era la de ganarse las simpatías (y el apoyo) de un país perteneciente a la Sociedad de las Naciones. Otro tema que se señala en el memorando es que Etiopía tenía una economía basada en la esclavitud, destacando cómo dos millones de esclavos vivían en el país, y otro millón era enviado anualmente hacia los países árabes, mientras la población vivía en un «estado de barbarie reconocida». El memorando se cerraba con la petición de que los representantes ecuatorianos en Ginebra se pusieran en contacto con los italianos, para que estos últimos pudieran aclarar el punto de vista de Italia antes de que Ecuador decidiera su postura de cara a las próximas reuniones en la Sociedad de las Naciones ${ }^{56}$. Es evidente que la propaganda italiana estaba enfocada a ganar el apoyo del país, presentándose a la vez como víctima y como potencia civilizadora, para dirigir Ecuador hacia una política contraria a las sanciones en el seno de la Sociedad.

Finalmente, el 3 de octubre Italia atacó a Etiopía: un país perteneciente a la Sociedad agredía a otro igualmente miembro. Comenzaba la guerra. Las sanciones fueron aprobadas con el voto favorable del representante de Ecuador, y estuvieron vigentes a partir del día 18 de noviembre.

De las palabras del ministro de Relaciones Exteriores de Ecuador se entiende perfectamente que el país andino no era un extremo defensor del frente favorable a las sanciones contra Italia (liderado por gran Bretaña), más bien parece que, por fin, había decidido votar de manera conforme a la mayoría de los otros estados pertenecientes a la Sociedad, pero sin estar completamente convencido de la utilidad de las sanciones. Afirma Chiriboga, en aquel entonces ministro de Relaciones Exteriores de Ecuador:

Al sumar su voto al de la mayoría, nuestro Representante en Ginebra hizo presente que las sanciones comprendidas y aplicadas según el espíritu del Pacto, no pueden ser consideradas como punitivas, ni contienen ningún elemento capaz de herir el amor propio de los pueblos que, a veces guiados por un impulso apasionado pero pasajero se lanzan a la guerra. Representan — dijo - tan solo un modo de presión para volver al camino del Pacto, a la Parte, que se ha extraviado, y asegurar la paz a la mayor brevedad posible ${ }^{57}$.

Por fin, parece que la obra de propaganda y sensibilización italiana del país andino había dado algunos resultados. Destaca que las relaciones diplomáticas entre Italia y Ecuador no fueron interrumpidas.

56 Memorándum que presenta el Sr. Ministro de Italia hoy 27 de agosto de 1935, 1935, AHMRE B.25.8. Legación de Italia en el Ecuador (1934-1935).

57 Chiriboga, 1936: 66. 
La cuestión de la aplicación de las sanciones a Italia fue objeto de debate en el país andino: por un lado, estaba la oposición política, que representaba el frente favorable a las sanciones; por otro lado, el gobierno estaba más preocupado por las repercusiones económicas negativas que dichas sanciones pudieran provocar en Ecuador (siendo Italia un socio económico importante en aquel momento) $)^{58}$.

Hay que destacar, además, que el sentimiento de amistad respecto a Italia, desarrollado en estas circunstancias por el gobierno, no iba a la par con el sentimiento de gran parte de la opinión pública ${ }^{59}$. Como se matiza en otros trabajos, precisamente en este periodo, la imagen de Italia, y de los inmigrantes italianos, que se tenía en Ecuador, empieza a cambiar volviéndose cada vez más negativa ${ }^{60}$, mientras que una parte considerable de la elite político-económica aún miraba con amistad hacia la península.

En todo caso, y de acuerdo a lo realizado por varios países latinoamericanos, Ecuador no aplicó las sanciones. Según lo afirmado por la diplomacia italiana en el país andino, en diciembre de 1935, frente a una reunión de «amigos políticos», admiradores y simpatizantes de la Italia de la época, el presidente Federico Páez Chiriboga había tomado el empeño formal de que en ningún caso iba a adherirse a la política sancionadora contra ella. Por esta razón el gobierno, al comienzo, tuvo una postura de incertidumbre o ambivalente, con la esperanza de que una rápida victoria italiana conllevara una definitiva resolución del problema de las sanciones. En marzo de 1936 pareció darse un cambio de actitud, acercándose el delegado en Ginebra hacia la línea de aplicación de las sanciones. Después de un intercambio — no exactamente claro- entre el gobierno y el delegado ecuatoriano, por fin Ecuador aclaró que había tardado en la aplicación de dichas sanciones y pensaba tener que proceder a su anulación ${ }^{61}$.

De manera pública y formalmente, a partir de marzo las sanciones ya no fueron aplicadas por Ecuador, «considerando la ineficacia de las sanciones para la cesación del conflicto» ${ }^{62}$. Luego, en el momento en el que las tropas

58 Dr. Renato Baccinetti, Cancelliere della R. Legazione, Legazione d'Italia in Quito, Relazione Politico economica per l'anno 1936, 1936, ASDMAE, Serie Affari Politici 19311945 Equatore busta 3 (1937).

59 Casimiro De Lieto, Legazione d'Italia in Quito, Oggetto: "Nostra situazione nella Repubblica dell'Equatore e possibilità di influenze-propaganda", Quito, 3 novembre 1936, ASDMAE, Serie Affari Politici 1931-1945 Equatore busta 3 (1937), prot $n^{\circ}$ 20067/213.

60 Pagnotta, 2016: 123 et seg.

61 Dr. Renato Baccinetti, Cancelliere della R. Legazione, Legazione d'Italia in Quito, Relazione Politico economica per l'anno 1936: 2-3.

62 Chiriboga, 1936: 68-69. 
italianas entraron en Adís Abeba (el 9 de mayo de 1936), el Gobierno ecuatoriano anunció otra vez que no secundaría por más tiempo la política de la Sociedad de las Naciones ${ }^{63}$. Fue el 4 de julio de 1936 cuando finalmente la Sociedad levantó las sanciones y demostró el importante fracaso internacional por la incapacidad de actuación en el conflicto.

Cabe destacar que los periódicos del país andino siguieron con atención la evolución de la guerra. El periódico quiteño El Comercio detalló los bombardeos y la utilización de los gases, prohibidos en los acuerdos internacionales, por parte de los italianos ${ }^{64}$, presentando los hechos de manera descriptiva. Es cierto que la fuente de muchas informaciones internacionales reportadas en el periódico era la agencia alemana de noticias Transocean, creada en 1915.

En todo caso, las pretensiones imperialistas de Italia, tal como se manifestaron durante y después de la guerra de Etiopía, preocuparon a muchos gobiernos extranjeros. A continuación, y gracias al progresivo alineamiento con la política de defensa continental puesta en marcha en la segunda guerra mundial, irá cambiando la actuación de los gobiernos locales latinoamericanos, entre otros el de Ecuador, hacia los países totalitarios y hacia los inmigrantes provenientes u originarios de ellos ${ }^{65}$. De una inicial simpatía, vista, por ejemplo, en el caso del viaje de la Nave Italia, y en la ambigüedad demostrada en la aplicación de las sanciones, se pasará a un progresivo rechazo.

\section{HOY EN DÍA ¿SON ORGULLOSOS Y FIELES LOS ITALIANOS EN EL EXTERIOR?}

Para conquistar la hegemonía política sobre los italianos presentes en Ecuador, conforme a lo que hizo en otros países, el fascismo desplegó sus labores hacia la creación de un sentimiento de adhesión al régimen que pudiera justificar el lema: «Sono oggi fieri e fedeli gli italiani all'estero» (Hoy en día son orgullosos y fieles los italianos en el exterior) ${ }^{66}$. Por lo que concierne a la propaganda más específicamente dirigida hacia los ciudadanos ecuatorianos y los inmigrantes italianos, cabe destacar que la Legación italiana se ocupaba de la difusión del material producido y enviado por el Minis-

\footnotetext{
63 "Felicita por el retiro de sanciones a Italia", El Comercio, 9 de mayo de 1936: 3

64 Varios artículos publicados en El Comercio de Quito entre el mes de octubre de 1935 y mayo de 1936.

65 Pagnotta, 2016: 139-169.

66 Lema reproducido en los muros de la Esposizione della Rivoluzione Fascista de 1932. Cit. in. Moure Cecchini, 2016: 448.
} 
terio de la Cultura Popular, y de la publicación de noticias y artículos pro-régimen en la prensa ecuatoriana.

De toda manera, precisamente en 1936, cuando era urgente la necesidad de ganar apoyos para el gobierno, a causa de la invasión de Etiopía y la consecuente pérdida de simpatías internacionales hacia Italia, los grupos italianos presentes en Quito y Guayaquil no parecían mostrar su adhesión al fascismo ni trabajaban a su favor, más bien destacaba su indiferencia o posición a-fascista. A este propósito, el cónsul destaca que

las colonias de Quito y Guayaquil no ejerciesen alguna acción política ${ }^{67}$. La de Quito [...] es poco numerosa, unas sesenta personas en total, compuesta por napolitanos en su mayoría, que prevalentemente comercian en tejidos, son acomodados, estimados, pero nada más. La de Guayaquil, mas considerable y numerosa, está compuesta por alrededor de un millar de personas, comerciantes con capitales más importantes, pero también ajena a cualquier influencia política ${ }^{68}$.

Por fin, los inmigrantes parecían mostrar un sustancial desinterés hacia la política del país de origen. Para incrementar la obra de propaganda, que pudiera esparcir sus semillas entre ellos, haciéndoles potenciales instrumentos de apoyo en el exterior, y para implementar, en general, el soporte internacional, resultó entonces importante bien ejercer una influencia hacia la prensa local, bien crear una prensa étnica, afín al régimen.

Por lo que concierne al primer caso, esa influencia tenía que basarse sobre la puesta en valor de la actividad y la política de la Italia fascista, «dirigiendo la mirada hacia Roma, capital de la latinidad [...] para dirigir en manera favorable la opinión pública hacia nosotros ${ }^{69}$. Sin lugar a duda, eso significaba negociar económicamente con los principales periódicos de Quito y Guayaquil, para que no publicasen noticias hostiles a la Italia fascista, más bien lo contrario $^{70}$.

Pero, solo un año después de expresada la afirmación anterior, la prensa del país era ya considerada genéricamente hostil a Italia; precisamente por esta razón se empezó a publicar el periódico La Idea Latina, con el fin de lograr destacar de manera autónoma la voz de Italia en el ambiente ecuatoriano. En el nombre de la revista (La Idea Latina) recurría nuevamente aquella idea de cercanía cultural, ideológica y espiritual entre Italia y Ecua-

67 Entendiéndose, aquí, de apoyo al fascismo.

68 Dr Casimiro De Lieto, Legazione d'Italia in Quito, Oggetto: "Nostra situazione nella Repubblica dell'Equatore e possibilità di influenze-propaganda".

69 Idem.

70 Idem. Las fuentes señalan que la diplomacia italiana pidió el dinero para proveer esta obra de propaganda, pero desconocemos si Italia efectivamente otorgó este fondo. 
dor bajo el emblema de la latinidad liderada por Italia fascista. La revista estaba financiada con mil sucres mensuales enviados por el Ministerio de la Cultura Popular, y cada número tenía una tirada de 1.200 ejemplares $^{71}$. También contribuían a su financiación el Banco Italiano, la Compañía de seguros 'Italia' de Lima y la Sociedad de navegación 'Italia'72. La idea latina estaba publicada en español, y era difundido prevalentemente entre los ecuatorianos «de algún nivel intelectual» y entre «los obreros más inteligentes al frente de las organizaciones [¿obreras?, ¿sindicales?] $\gg^{73}$. La tentativa de ganar consenso entre los ecuatorianos que revestían cargos de peso en el mundo laboral es evidente, aunque, otra vez, los resultados parecen escasos.

Finalmente, el 1 de julio de 1939 se suspendió la publicación, por la reducción drástica del financiamiento del Ministerio de la Cultura Popular, y porque, además, esta otra experiencia en el mundo de los medios de comunicación en Ecuador no parecía tener gran éxito tampoco entre los italianos, remarcando, otra vez, un desinterés de fondo de la mayoría de los inmigrantes hacia los intereses o planes de la Italia fascista ${ }^{74}$.

En todo caso, es evidente que el régimen quiso jugar un papel preeminente en la representación de los italianos en el país, tratando, por ejemplo, de apoderarse de las antiguas organizaciones recreativas y mutualistas que los inmigrantes se habían dado. Un caso peculiar es el de la Società di beneficenza per gli italiani 'Garibaldi'. Esa sociedad había sido fundada en 1882 en Guayaquil. La finalidad de dicha asociación era la de «cimentar la unión entre los miembros de la colonia y socorrer de la desgracia y la indigencia a todos los italianos que el Consejo de Administración considere dignos de

71 Anselmo Anselmi, Regio Consolato d'Italia in Guayaquil, Telespresso $n .1774$ indirizzato alla R. Legazione d'Italia, Oggetto: "Editorial Latina” — Vendita macchine tipografiche. Riferimento: Telespresso di codesta R. Legazione n. 2808, Guayaquil, addì 1 dicembre 1939, anno XVIII, ASDMAE, Serie Affari Politici 1931-1945 Equatore busta 6 (1939).

72 Federico Saporiti, Legazione d'Italia in Quito, A sua Eccellenza Grand. Uff. Giovanni Amadori R. Ministro d'Italia Quito, Guayaquil 7 novembre 1939, XVIII, ASDMAE, Serie Affari Politici 1931-1945 Equatore busta 6 (1939).

73 Anselmo Anselmi, Regio Consolato d'Italia in Guayaquil, Telespresso n.1723 indirizzato alla R. Legazione d'Italia, Oggetto: Giornale "Idea Latina”, Riferimento: telespesso di cod. Legazione n.2570, Guayaquil, addì, 19 novembre 1939, XVIII', ASDMAE, Serie Affari Politici 1931-1945 Equatore busta 6 (1939), p. 1.

74 G. Amadori, R. Legazione d'Italia, al Regio Ministero degli Affari Esteri, Dir. Gen. Affari Trasoceanici e per conoscenza R. Ministero della Cultura Popolare, Posizione Equatore 34-1, Oggetto: "L'idea Latina", Riferimento: Telespresso Ministeriale 22390N22 in data 22 luglio sc., Quito, addì 28 ottobre 1939, anno XVIII, ASDMAE, Serie Affari Politici 19311945 Equatore busta 6 (1939). 
socorro» ${ }^{75}$. En el momento de la fundación, contaba con 108 socios, la mayoría pertenecientes a la elite étnica y comercial.

En las elecciones de 1928 se afirma que fue finalmente elegido un directorio fascista ${ }^{76}$. Más tarde, a pesar del trabajo desarrollado por la propaganda, en la tentativa de tomar el liderazgo de las viejas estructuras mutualistas, en 1930 la Sociedad eligió un director de signo político contrario. Este director era:

perteneciente a la masonería, y, a pesar de sus esfuerzos por no hacerlo evidente, de principios antifascistas, es de sentimientos patrióticos. Él, en todo caso, siempre se ha mostrado deferente hacia este consulado, y en cada ocasión, ha puesto a disposición, y sin dudarlo, los locales de la 'Garibaldi', como hizo para la proyección del film 'fascista' 'El grito del águila', a cuya proyección, sin embargo, él no asistión7.

Esas palabras identifican claramente la orientación ideológica del director de la sociedad italiana y, por ende, del grupo que acababa de elegirlo en aquel papel. Como afirma el mismo cónsul, se trata de un a-fascismo ejercido prevalentemente por los miembros más antiguos del grupo y que siempre habían liderado la 'Garibaldi' (aunque ya en ese momento algunos fascistas sean miembros de dicha organización $)^{78}$. Es cierto que, por otro lado, y por los documentos que hemos podido consultar, muchas de las celebraciones oficiales italianas fascistas se desarrollaron en la 'Garibaldi'. Hay que añadir que, a finales de los años treinta, se establecieron las bases para la creación de la Dante Alighieri en Ecuador ${ }^{79}$.

En el mismo período, De Lieto, recién instalado como ministro de la Legación, para proceder a una más efectiva fascistización, pensó en sustituir la

75 Statuto della Società italiana di beneficenza Garibaldi, instituita in Guayaquil il 24 giugno 1882, 1882: I.

${ }^{76}$ Al Ministero degli Affari Esteri, dal Regio Consolato d'Italia, 26 gennaio 1929 - VII , Protocollo n. 128 A 1, Guayaquil (Ecuador), 21, 26 gennaio 1929 - VII ${ }^{\circ}$, ASDMAE, Serie Affari Politici 1919-1930 pacco 1013 Equatore (1919-1926).

77 Al Ministero degli Affari Esteri, Roma, Il Regio Console Gino Busi, prot. $n^{\circ} 1394$ Al, A. VIII, 28 Maggio 1930, ASDMAE, Serie Affari Politici 1919-1930 pacco 1014 Equatore (1927-1930) [informe sin número de página].

78 Al Ministero degli Affari Esteri, Roma, Il Regio Console Gino Busi, 28 Maggio 1930.

79 Legazione d'Italia, al R. Ministero degli Affari Esteri Roma, Quito, telespresso n. 219/27, Riservato, Oggetto: Propaganda, Riferimento: suo telespresso circolare n.72, Quito, 22 gennaio 1938, Anno XVI, ASDMAE, Serie Affari Politici 1931-1945 Equatore busta 4 (1938), p. 2. La Società Dante Alighieri fue fundada a finales del XIX siglo como institución cultural destinada a difundir y tutelar la cultura italiana en el mundo y, en el periodo de nuestro interés, se acercaba a las posiciones político-culturales del fascismo. 
Garibaldi, subsumiéndola en la Casa de los Italianos ${ }^{80}$. Su idea no tuvo seguimiento, aunque por el momento desconocemos la razón.

Se hace evidente aquí una de las grandes diferencias entre el fascismo en el exterior y el fascismo en la patria. Mientras el fascismo en Italia había procedido a la eliminación de todas las demás organizaciones, en Ecuador, en el año 1930, el mismo cónsul sugería dejar que la Garibaldi fuera liderada por esos viejos italianos a-fascistas, para dejarles «un escape» y para que, con ese arreglo, fueran más sensibles a la propaganda fascista en otras circunstancias de mayor interés para la diplomacia del régimen ${ }^{81}$. Además, especifica el cónsul que esa convivencia es posible siempre que no damnifique la obra del consulado. Destaca aquí, y en el exterior más en general, la maleabilidad de la política fascista según el contexto en el que actuaba. En todo caso, la primera mitad de los años treinta en Ecuador se caracterizan por la coexistencia entre organizaciones fascistas y no-fascistas.

\section{EL DEBATE ACERCA DE UN HOMENAJE ROMANO}

El debate acerca del monumento a la amistad entre Italia y Ecuador nos permite distinguir entre la retórica del régimen y las efectivas adhesiones a la ideología de la patria de origen.

A comienzos de los años treinta, el prefecto de Guayaquil había dado nombres de colonias extranjeras a distintas partes del malecón, y había apelado a todas ellas para que contribuyeran en adornarlo. Ya habían procedido con el homenaje las colonias china y americana ${ }^{82}$. Por lo que concierne a los italianos, después de una genérica aceptación de la propuesta hecha por el prefecto, y tras una evaluación de las posibilidades de realización local para llevar a cabo la obra, que resultó negativa, los miembros indicados como «mejores» de la colonia pidieron la intervención del consulado para seleccionar la obra, y este último pidió la ayuda del Ministerio de Relaciones Exteriores Italiano ${ }^{83}$.

El famoso escultor italiano Attilio Selva entró en el debate en enero de 1932, proponiendo ceder para ello la muy conocida estatua 'Enigma' por él $219 / 27$.

${ }^{80}$ Legazione d'Italia, al R. Ministero degli Affari Esteri Roma, Quito, telespresso $n$.

81 Al Ministero degli Affari Esteri, Roma, Il Regio Console Gino Busi, 28 Maggio 1930.

82 Giuseppe Sapuppo, Legazione di S.M il Re d'Italia in Equatore, A sua Eccellenza Ministro Dino Grandi, Ministro degli Affari Esteri, prot. $n^{\circ}$ 1030/165. Quito, 15 agosto 1931 IX, ASDMAE, Serie Affari Politici 1931-1945 Equatore busta 1 (1931-1934), p. 1.

83 Giuseppe Sapuppo, Legazione di S.M il Re d'Italia in Equatore, A sua Eccellenza Ministro Dino Grandi, p. 2. 
realizada ${ }^{84}$. La propuesta no tuvo aceptación. Desde Roma llegaron disposiciones al Consulado para hacer «un oportuno trabajo de persuasión», de manera que los italianos más notables del puerto ecuatoriano homenajearan a la alcaldía de Guayaquil con la donación de una estatua ${ }^{85}$. Aquí parece entreverse un interés de la diplomacia y del gobierno italiano para que los inmigrantes demostraran también su amor a Ecuador, mientras estos mismos mostraban tan poco interés en esto como en satisfacer los pedidos que provenían de Roma. Las respuestas de los italianos de la ciudad portuaria no fueron para nada favorables, ya que los gastos previstos (alrededor de 20.000 sucres) parecían demasiado elevados. En segundo lugar, el sentimiento de hostilidad hacia los extranjeros que dominaba en aquel momento, y que hacía que se remplazaran técnicos y expertos italianos por locales, tampoco favorecía la realización de tal donación ${ }^{86}$.

El Dr. Anselmi, Reggente del Consulado de Guayaquil, dejó entrever la posibilidad de que los connacionales pudieran estar más interesados en caso de que la donación no fuera un homenaje a la ciudad de Guayaquil, sino más bien una expresión en Guayaquil del arte nacional italiano ${ }^{87}$. No podemos saber exactamente sobre qué bases el cónsul italiano hizo esta especulación. En todo caso, parece poderse deducir que no había un particular apego ni a la ciudad de llegada, ni a conformarse con los pedidos del régimen del país de partida. En aquel momento, la propuesta no tuvo seguimiento.

En 1936, nuevamente se volvió a hablar del tema, pero el objeto del homenaje era una histórica piedra romana. Impulsaba el debate el miembro del fascio, nacido en Ecuador, Ernesto Parodi. Ahora, además de manifestar la gratitud a Ecuador por no haber aplicado de hecho las sanciones a Italia dictadas por la Sociedad de las Naciones ${ }^{88}$ y a la ciudad de Guayaquil por haberlas rechazadas tempranamente ${ }^{89}$, la idea era situar la histórica piedra ro-

84 Bajo la compensación de 42.000 liras de la época, más o menos 43.800 euros actuales. Fuente de la equivalencia: http:/www.infodata.ilsole24ore.com/2016/05/17/calcola-poteredacquisto-lire-ed-euro-dal-1860-2015/. Consultado el 11/08/2017. Actualmente la estatua original se encuentra en la Galleria Nazionale d'Arte Moderna de Roma.

85 Giuseppe Sapuppo, Legazione di S.M il Re d'Italia in Equatore, Al R. Ministero degli Affari Esteri, telespresso $n^{\circ}$ 158/35 pos.C.13, Quito, 19 febbraio 1932 X, ASDMAE, Serie Affari Politici 1931-1945 Equatore busta 1 (1931-1934), p. 1.

86 Idem.

87 Idem.

88 Casimiro De Lieto, R. Legazione d'Italia in Equatore, Al R. Ministro degli Affari Esteri, oggetto: Masso romano a Guayaquil, Quito, 30 settembre 1937 XV, ASDMAE, Serie Affari Politici 1931-1945 Equatore busta 3 (1937), p. 2.

89 Ernesto Parodi, a S.E. l'On. Cav. Benito Mussolini, Sanremo, 22 maggio 1936 XIV, ASDMAE, Serie Affari Politici 1931-1945 Equatore busta 5 (1938). 
mana en una parte del malecón que el fascio, junto con el grupo italiano, preveía arreglar ${ }^{90}$. El mismo Mussolini parece que se había expresado en relación con ello para favorecer y desarrollar las relaciones con Ecuador precisamente en agradecimiento por la postura mantenida durante la guerra de Etiopía ${ }^{91}$.

Nuevamente, los altos gastos previstos (alrededor de 55.000 liras) para la realización del homenaje dieron lugar a protestas por parte de los residentes italianos en Guayaquil que supuestamente tenían que asumirlos. Estos, además, hicieron constar que no tenían la menor intención de sacrificarse para arreglar parte alguna del malecón. Destaca De Lieto que entre los opositores al proyecto se encontraban los mismos hermanos de Ernesto Parodi ${ }^{92}$. El mismo De Lieto actuó para convencer al Gobierno de que era mejor una solución local, menos onerosa y de un gasto de 15.000 liras, para poder dirigir otros fondos hacia otros proyectos que él tenía más en consideración (como el proyecto de la revista italiana, el del Instituto Italiano de cultura, el de la Casa de los Italianos) y resultarían más útiles, en su opinión, para construir una amistad más duradera respecto a la Italia fascista ${ }^{93}$. Destaca siempre De Lieto que el grupo de Guayaquil tampoco fue muy entusiasta de la perspectiva de gastar esta cifra reducida ${ }^{94}$. Por encima del escaso entusiasmo del grupo italiano en Guayaquil por realizar este homenaje, el responsable de la Legación, Casimiro De Lieto, pensaba que a mediados de los años treinta, en los tiempos en que se encontraban, necesitaban ya proceder a una verdadera y duradera obra de fascistización del grupo italiano, que hasta aquel momento no se había alcanzado. Esa obra se tenía que orientar hacia la reorganización y el fortalecimiento patriótico, más que a invertir sus esfuerzos en ese homenaje, cuyos resultados serían, estaba convencido de ello, efímeros ${ }^{95}$.

El ministro Ciano volvió a insistir en el homenaje, y apareció entonces la propuesta de que fuera hecho al Gobierno de Ecuador - y no solo a la ciudad

90 Galeazzo Ciano, all'On. Dott. Ernesto Parodi, Deputato al Parlamento Nazionale, Roma, 17 luglio 1936 XIV, ASDMAE, Serie Affari Politici 1931-1945 Equatore busta 5 (1938).

91 F. Cortese. Ministero degli Affari Esteri, al r. Ministero per la Stampa e la Propaganda, oggetto: Proposta ricordo antica Roma all'Equatore, telespresso $n^{\circ} 227425 / 548,13$ agosto 1936 XIV, ASDMAE, Serie Affari Politici 1931-1945 Equatore busta 5 (1938).

92 Casimiro De Lieto, R. Legazione d'Italia in Equatore, Al R. Ministro degli Affari Esteri, oggetto: Rapporti fra Italia ed Equatore, Quito, 13 luglio 1937 XV, ASDMAE, Serie Affari Politici 1931-1945 Equatore busta 5 (1938)

93 Idem.

94 Idem.

95 Idem. En el apartado anterior ya hemos dibujado sus propósitos y sus logros acerca de la revista, la casa italiana y el instituto de cultura italiana. 
de Guayaquil—, así como por parte de todos los italianos residentes en el país andino, y que fuera el Gobierno local quien decidiera dónde ubicar la donación «del gobierno fascista» ${ }^{96}$. Además, el ministro de Educación Nacional propuso que dicha piedra histórica fuera un bloque de las murallas de Roma del IV siglo a.C. Según este, el bloque tenía que ser una piedra, decorada con ornamentos de bronce a expensas del fascio local ${ }^{97}$.

Finalmente, en septiembre de 1937, De Lieto subrayó que ese homenaje no iba a destacar de manera prominente sobre los otros regalos entregados por parte de otros grupos nacionales, añadiendo además que la entrega de una piedra histórica romana no sería debidamente apreciada en el país andino. El diplomático parece que no estaba satisfecho con la poca adhesión a estos proyectos manifestada por los italianos de Guayaquil, y resaltó que solo después de dos meses de trabajos de persuasión, los italianos de Guayaquil habían accedido a cubrir los gastos para la piedra histórica. En conclusión, aconsejó postergar la entrega del homenaje al momento en el que la obra de fascistizacion estuviera ya cumplida, para que se diera así a esa entrega el relieve que merecía ${ }^{98}$. No tenemos noticias posteriores.

\section{CONSIDERACIONES FINALES}

En este artículo se ha pretendido contribuir a interpretar la relación trasnacional entre migrantes, el país de origen, y el de llegada, a partir del estudio del caso del proceso de inmigración y de las relaciones entre Italia y Ecuador en los años del fascismo. Un énfasis particular se da al estudio de este fenómeno a través un análisis sobre la propaganda fascista dirigida, por un lado, a los sectores gubernamentales y diplomáticos ecuatorianos; por otro, a la sociedad local más en general; y, por otro, a los inmigrantes italianos residentes en el país andino.

Se ha mostrado, en la primera parte, que el interés por la realización de políticas de lo que se denominó «un imperialismo pacífico», en Ecuador, tiene sus raíces en la Italia liberal, y conoció un renovado vigor por obra del

\footnotetext{
96 Galeazzo Ciano, Roma, 17 luglio 1936 XIV, all'On. Dott. Ernesto Parodi, Deputato al Parlamento Nazionale.

97 Cesare Maria de Vecchi di Val Cismon - Ministero dell'Educazione Nazione al R. Ministero degli Affari Esteri, oggetto: proposta dono ricordo o vestigia antica Roma all'Equatore, 3 luglio 1936, ASDMAE, Serie Affari Politici 1931-1945 Equatore busta 5 (1938).

98 Casimiro De Lieto, R. Legazione d'Italia in Equatore, Al R. Ministro degli Affari Esteri, oggetto: Masso romano a Guayaquil, p. 3.
} 
régimen fascista, que trató de convertir a los inmigrantes italianos en agentes de su política expansiva y de propaganda. En este sentido, la llegada de la Nave Italia a Ecuador fue el momento más representativo de esta tentativa de colonización pacifica, bajo el lema de la «latinidad» — de la que Roma era el faro- y que venía de la mano con una penetración económico-comercial por parte de las industrias italianas (incluyendo las bélicas).

Se ha destacado, también, cómo las tentativas de penetración y de propaganda en el país andino se hicieron más contundentes como consecuencia de la guerra de Etiopía, ya que, en ese momento, el régimen necesitaba recuperar consenso y simpatías hacia su obra, y más preeminentes se hicieron todavía las tentativas de fascistizacion de las estructuras italianas en tierra ecuatoriana, por lo que hemos visto, con escasos resultados. Si, de un lado, la diplomacia ecuatoriana actuó en el ámbito internacional de manera ambigua, y sin aplicar de hecho las sanciones, mostrando así una cercanía con la hermana latina, y quizá un cierto éxito de la propaganda fascista, bien diferente fue el resultado de la propaganda entre los inmigrantes italianos.

Cabe matizar que en este texto se ha manifestado la distancia entre los discursos ideológicos sobre el vínculo con el país de origen, y la realidad factual en la que se encontraron muchos inmigrantes. Si para el fascismo los connacionales estaban todos orgullosos y eran fieles al régimen, queda claro que en el Ecuador de los años treinta se asiste a un general desinterés por las políticas de la madre patria, o, mejor dicho, a un a-fascismo de base.

Al contrario, donde la propaganda del régimen italiano tuvo más acogida no fue entre la mayoría de los inmigrantes, sino más bien entre las élites (diferentes) que se alinearon con el poder en Ecuador durante los veinte años en que el fascismo se mantuvo en el poder. Se han mostrado los heterogéneos intereses económicos, geopolíticos y comerciales que conectaban a los grupos de poder en Ecuador e Italia hasta la víspera de la segunda guerra mundial.

\section{BIBLIOGRAFÍA}

Aliprandi, Ermenegildo y Martini, Virgilio, Gli italiani in Equatore, Rassegna delle vite e delle opera della stirpe italica in terra ecuatoriana, Guayaquil, Artes Gráficas 'Senefelder', 1930a.

Aliprandi, Ermenegildo y Martini, Virgilio, Anuario Ecuatoriano, Guayaquil, Editores Apartados 1107, 1930b. 
Almeida, Mónica, "Los sirio-libaneses en el espacio social ecuatoriano: cohesión étnica y asimilación cultural", Journal de la Société des Americanistes, 83 (Paris, 1997): 201-227.

Ayala Mora, Enrique, (ed.), Nueva Historia del Ecuador. Vol. X: Época Republicana IV, Quito, Corporación Editora Nacional, 1983.

Ayala Mora, Enrique, Resumen de Historia del Ecuador, Quito, Corporación Editora Nacional, 2008.

Bejarano, Ingrid. "La emigración árabe al Ecuador", Anaquel de Estudios Árabes, VIII (Madrid, 1997): 57-70.

Bertonha, João Fábio, Sob a sombra de Mussolini: os italianos de São Paulo e a luta contra o fascismo, 1919-1945, São Paulo, FAPESP/Annablume, 1999.

Bertonha, João Fábio, "Emigrazione e politica estera: la 'diplomazia sovversiva' di Mussolini e la questione degli italiani all'estero, 1922-1945”, Altreitalie, 23 (Torino, 2001a): 39-60

Bertonha, João Fábio, O fascismo e os imigrantes italianos no Brasil, Porto Alegre, Edipucrs, 2001b.

Bianchi, Ornella, "Fascismo ed emigrazione", Vanni Blenghino (ed.), La riscoperta delle Americhe. Lavoratori e sindacato nell'emigrazione italiana in America Latina, 1870-1970, Milano, Nicola Teti Editore, 1994: 96-114.

Bustos, Guillermo, "La fragilidad de las huellas de la memoria y la 'incuria' en el manejo de los archivos históricos en Ecuador", Santiago Cabrera Hanna (ed.), Patrimonio cultural, memoria local y ciudadanía. Aportes a la discusión, Quito, Universidad Andina Simón Bolívar/Corporación Editora Nacional, 2011: 51-64.

Cannistraro, Philip y Gianfausto, Rosoli, "Fascist emigration policy in the 1920's: an interpretative framework", International Migration Review, $13 / 4$ (Hoboken, 1979): 673-692

Chiriboga, Ángel Isaac, Informe que el Ministro de Relaciones Exteriores presenta a la Nación. Julio de 1935 - Septiembre de 1936, Quito, Taller Tipográfico Nacional, 1936.

Ciccarelli, Orazio, "Fascist propaganda and the Italian community in Peru during the Benavides regime, 1933-39", Journal of Latin American Studies, 20/2 (London, 1988): 361-388.

Crawford de Roberts, Louis, El Ecuador en la época cacaotera. Respuestas locales al auge y colapso en el ciclo mono exportador, Quito, Editorial Universitaria, 1980.

Crawford, Louise, Los libaneses en el Ecuador. Una vida de éxitos, Quito, Imprenta Segura, 1996. 
De Caprariis, Luca, "I Fasci italiani all'estero", Emilio Franzina y Matteo Sanfilippo (eds.), Il fascismo e gli emigranti, Milano, Laterza, 2003: 3-26.

De Felice, Renzo, Mussolini il duce. Vol. I: Gli anni del consenso, 1929-1936, Torino, Einaudi, 1974.

Di Capua, Alberto, "Brevi cenni sull'emigrazione di ebrei italiani in Ecuador (19381945)", Eleonora Maria Smolensky y Vera Vigevani Jarach, Tante voci, una storia. Italiani ebrei in Argentina 1938-1948, Bologna, Il Mulino, 1998: 433-449.

Embajada de Italia (Quito), (ed.), Los Italianos y el País de la Mitad del Mundo, Quito, Ediecuatorial, 2008.

Estrada, Jenny, Los españoles de Guayaquil, Guayaquil, Sociedad Española de beneficencia, 1992.

Estrada, Jenny, Los italianos de Guayaquil. Guayaquil, Societá di Assistenza Italiana "Garibaldi", 1993.

Finchelstein, Federico. Transatlantic Fascism: Ideology, Violence, and the Sacred in Argentina and Italy, 1919-1945, Durham and London, Duke University Press, 2010.

FitzGerald, David y Cook-Martín, David, Culling the Masses. The Democratic Origins of Racist Immigration Policy in the Americas, Cambridge (Massachusetts) \& London, Harvard University Press, 2014.

Foote, Nicola y Goebel, Michael, (eds.), Immigration and National Identities in Latin America, Gainesville, University Press of Florida, 2014.

Franzina, Emilio y Sanfilippo, Matteo, (eds.), Il fascismo e gli immigrati, Bari, Laterza, 2003.

Furfaro, Domenico, "Il contributo di Vincenzo Grossi al dibattito sul problema della colonizzazione e dell'emigrazione in occasione del secondo Congresso Geografico Italiano", Miscellanea di Storia delle Esplorazioni VII, Genova, Buozzi, 1982: 203-245.

Gentile, Emilio, "La politica estera del partito fascista. Ideologia e organizzazione dei Fasci italiani all'estero", Storia Contemporanea, XXVI/6 (Bologna, 1995): 897-956.

Grubel Rosenthal, Manuel, Ecuador destino de migrantes. Una biografia de la comunidad judía del Ecuador, Quito, PPL Impresores, 2010.

Guarnieri Calò Carducci, Luigi, Dizionario storico-biografico degli italiani in Ecuador ed in Bolivia, Bologna, Il Mulino, 2001.

Kreuter, María-Luise, ¿Dónde queda el Ecuador? Exilio en un país desconocido desde 1938 hasta fines de los años cincuentas, Quito, Abya-Yala, 1997.

La nave 'Italia' en el Ecuador. Recuerdo de la visita de la Embajada italiana y su séquito. La muy noble y muy leal ciudad de San Francisco de Quito - Agosto 1924, Quito, Francisco \& Páez, 1924. 
Lesser, Jeffrey, Immigration, Ethnicity and National Identity in Brazil, 1808 to the Present, New York, Cambridge University Press, 2013.

Luconi, Stefano, La 'diplomazia parallela'. Il regime fascista e la mobilitazione politica degli italo-americani, Milano, Franco Angeli, 2000.

Luconi, Stefano y Guido, Tintori, L’ombra lunga del fascio. Canali di propaganda fascista per gli 'italiani d'America', Milano, M\&B Publishing, 2004.

Ministerio de Economía - Dirección General de Estadística y Censos, Primer Censo de Población del Ecuador. Vol. IV - Tomo I: Población por idiomas y dialectos, Quito, Talleres Gráficos de la Dirección, Quito, 1950.

Moure Cecchini, Laura, "The Nave Italia and the Politics of Latinità: Art, Commerce, and Cultural Colonization in the Early Days of Fascism", Italian Studies, 71/4 (London, 2016): 447-476. http://doi.org/10.1080/00751634.2016.1222755

Ostuni, Maria Rosaria, "Leggi e politiche di governo nell'Italia liberale e fascista", Pietro Bevilacqua, Andreina De Clementi y Franzina Emilio (eds.), Storia dell'emigrazione italiana. Partenze, Roma, Donzelli, 2001: 309-321.

Pagnotta, Chiara, Situando los márgenes de la Nación. Los italianos en Ecuador (XIX-XX), Quito, Abya-Yala/TEIAA, 2016.

Ponce, Nicolás Clemente, Informe del Ministerio de Relaciones Exteriores. Junio de 1923, Junio de 1924, Quito, Taller Tipográfico Nacional, 1924.

Ponce Borja, Alejandro, Informe que el Ministro de Relaciones Exteriores presenta a la Nación. Junio de 1934 - Junio de 1935, Quito, Taller Tipográfico Nacional, 1935.

Poupalt, Christopher, "Les voyages d'hommes de lettres en Italie fasciste: Espoir du rapprochement franco-italien et culture de la latinitè", Vingtième Siécle. Revue d'Histoire, 4/104 (Paris, 2009): 67-84.

Pretelli, Matteo, "La risposta del fascismo agli stereotipi degli italiani all'estero", Altreitalie, 28 (Torino, 2004): 48-66.

Pretelli, Matteo, "Il fascismo e l'immagine dell'Italia all'estero", Contemporanea, XI/2 (Bologna, 2008): 221-242.

Quintero, Rafael y Silva, Erika, Ecuador: una Nación en Ciernes, Vol. I, Quito, Editorial Universitaria, 2001, tomo I. [Primera edición: Quito, FLACSO — Abya-Yala, 1991].

Ramírez, Jacques, (ed.), Ciudad-Estado. Inmigrantes y políticas. Ecuador, 18901950, Quito, IAEN, 2012.

Roca, Octavio S., Informe que eleva al Señor Ministro del Interior y Policía a la Intendencia de la Provincia del Guayas correspondiente al año de 1899, Guayaquil, Tipografía 'El Vigilante', 1900. 
Saint Geours, Yves, "La Sierra Centro y Norte. (1830-1925)", Juan Maiguashca (ed.), Historia y Región en el Ecuador, Quito, FLACSO/Corporación Editora Nacional, 1994: 143-188.

Scarzanella, Eugenia y Trento, Angelo, "L'immagine dell'America Latina nel fascismo italiano", Agostino Giovafnoli y Giorgio Del Zagna (eds.), Il mondo visto dall'Italia, Milano, Edizioni Angelo Guerini, 2004: 217-227.

Soave, Paolo, La «scoperta» geopolitica dell'Ecuador. Mire espansionistiche dell'Italia ed egemonia del dollaro 1919-1945, Milano, Franco Angeli, 2008.

Statuto della Società italiana di beneficenza Garibaldi, instituita in Guayaquil il 24 giugno 1882, Guayaquil, Imprenta de 'La Nación', 1882.

Trento, Angelo, "«Dovunque è un italiano, là è il tricolore». La penetrazione del fascismo tra gli immigrati in Brasile", Eugenia Scarzanella (ed.), Fascisti in Sud America, Firenze: Casa Editrice Le lettere, 2005: 1-54.

Yankelevích, Pablo, (ed.), Inmigración y racismo. Contribuciones a la historia de los extranjeros en México, México, Colegio de México, 2015.

Fecha de recepción: 3 de enero de 2018.

Fecha de envío de las modificaciones: 8 de mayo de 2018.

Fecha de aceptación: 9 de julio de 2018.

\section{Ecuador, Immigrants and Fascist Propaganda (1922-1938)}

This text focuses on fascist propaganda in Ecuador, directed either at public opinion and the Ecuadorian government, or at Italians living in Ecuador in the period between Mussolini's power grab in Italy (1922) and the eve of the Second World War (1938). It seeks to analyse immigration from a totalitarian country, in a bid to explain two main points: firstly how fascism relates to Ecuadorian society, and secondly, the 'mechanisms of consensus' spread by propaganda, their performance and achievements (or otherwise) among Italian immigrants, the elite and Ecuadorian society at that time. The study will be based on documentation from the Historical Diplomatic Archive in Rome and the Alfredo Pareja Diezcanseco Historical Archive of the Ministry of Foreign Affairs in Quito.

Key words: Ecuador; Italy; Fascism; Propaganda; Immigrants; Ecuadorian Society. 